\title{
Diritti umani, tutela della salute e sicurezza dei lavoratori e responsabilità sociale d'impresa: Quali prospettive?
}

\author{
Michela Cavallini \\ Emile, Italian \\ michela.cavallini@unimore.it \\ Scuola di dottorato in \\ Relaxioni di Lavoro, \\ Universitá degli Studi di \\ Modena e Reggio
}

Recebido em: 12/11/2010

Aprovado em: 24/03/2011

\section{Resumo}

O reconhecimento da saúde e segurança dos trabalhadores como direito humano é essencial para assegurar a proteção universal. O tema é, portanto, examinado em relação ao direito internacional dos direitos humanos, em relação as políticas mais recentes de Organização Internacional do Trabalho e segundo a perspectiva da Carta Social Europeia. Levando em conta também uma série de questão do direito do trabalho italiano, é necessário ter em conta o quadro jurídico e promover a cooperação entre os diferentes atores envolvidos, para verificar a validade e garantir a eficácia das políticas de Corporate Social Responsibility.

\section{Palavras-chave}

Saúde e segurança. Direitos Humanos. Responsabilidade social da empresa. 


\title{
Human rights, protection of the health and safety of workers and corporate social responsibility: What future prospects?
}

\author{
Michela Cavallini
}

Abstract

The recognition of health and safety of workers as a human right is central for granting its universal protection. The issue is therefore examined in relation to international buman rights law, International Labor Organization most recent policies and under the perspective of the Revised European Social Charter. Taking also into account some issues arisen in Italian labour law, we maintain that it is necessary to take into consideration the relevant legal context and to foster cooperation among different actors involved for assessing validity and granting effectiveness to Corporate Social Responsibility practices.

Key words

Health and safety. Human rights. Corporate social responsibility. 


\section{Sumário}

1 Introduzione

2 Il diritto internazionale dei diritti umani e

la salute e sicurezza dei lavoratori:

La Dichiarazione Universale dei Diritti dell'Uomo

e il Patto Internazionale sui diritti economici, sociali e culturali

3 L'Organizzazione Internazionale del Lavoro:

il ruolo della salute e sicurezza nelle politiche più recenti

4 Il Consiglio d'Europa e i diritti economici e sociali:

la Carta Sociale Europea.

5 Quali spunti di riflessione nel diritto del lavoro italiano?

6 Quale ruolo per la Responsabilità Sociale d'Impresa?

7 Osservazioni conclusive.

Bibliografia 


\section{Introduzione}

La garanzia di condizioni di lavoro sane e sicure gode di una posizione di rilievo nel contesto dei diritti e delle tutele proprie dei lavoratori. Di conseguenza, l'assicurazione non solo dell'efficacia giuridica, ma anche dell'effettività delle tutele e l'irrilevanza di qualsiasi argomentazione puramente economica volta a ridurne l'ambito di applicazione costituiscono oggetto di riflessioni e di argomentazioni di primaria importanza.

A questo proposito, l'analisi della tutela della salute e sicurezza sul lavoro nel contesto più ampio dei diritti umani ${ }^{1}$, dei diritti fondamentali dei lavoratori e delle pratiche di Responsabilità Sociale d'Impresa $(\mathrm{RSI})^{2}$, può offrire a nostro parere utili spunti nel contesto di una riflessione che si pone lo scopo di individuare gli effetti positivi derivanti dalla relazione tra questi tre ambiti.

La garanzia di condizioni di lavoro decenti (o dignitose, a seconda di come si preferisca tradurre il termine inglese decent), che tutelino, tra gli altri aspetti, la salute e la sicurezza dell'individuo, è un diritto inalienabile di chiunque svolga un'attività lavorativa, nonché la base per uno sviluppo economico sostenibile nei confronti delle persone e dell'ambiente ${ }^{3}$.

Questa dimensione di universalità e di inalienabilità è tuttavia messa in continua discussione, a livello globale, dall'impiego di lavoratori nei settori considerati più a rischio quali l'agricoltura, l'industria del legno, della pesca, delle costruzioni; dalla presenza di ampi contesti di economia sommersa; dalla dimensione di genere e dal lavoro di minori e di migranti; dalla diffusione di pratiche di violenza e di molestie nei luoghi di lavoro ${ }^{4}$.

1 I diritti umani, vale la pena ricordarlo brevemente, spettano ad ogni essere umano in quanto tale e sono dotati di caratteristiche peculiari: essi sono uguali per tutti gli esseri umani, inalienabili e universali. Con riferimento all' art. 5 della Dichiarazione di Vienna adottata nell'ambito delle Nazioni Unite nel 1993, tutti i diritti umani, al di là della loro qualificazione come diritti civili, politici, economici o sociali, sono universali, indivisibili, interdipendenti ed interconnessi. Cf DONNELLY, J. Universal Human Rights in theory and practice, Ithaca: Cornell University Press, 2002. p. 7, 10 e 27.

2 La Responsabilità Sociale d'Impresa viene definita, all'interno dell'Unione Europea, come "l'integrazione volontaria delle preoccupazioni sociali ed ecologiche delle imprese nelle loro operazioni commerciali e nei loro rapporti con le parti interessate" attraverso, ad esempio, "l'applicazione di norme sociali che superano gli obblighi giuridici fondamentali". Cf COMMISSIONE DELLE COMUNITÀ EUROPEE. Libro Verde Promuovere un quadro europeo per la responsabilità sociale delle imprese. $\operatorname{COM(2001)} 366$ def, Bruxelles: 2001. p. 7

3 INTERNATIONAL TRADE UNION CONFEDERATION (ITUC). $2^{\text {nd }}$ World Congress Resolution on extending social protection and ensuring good occupational health and safety. 2CO/E/6.12 (final). Vancouver: 2010. p. 3

4 INTERNATIONAL TRADE UNION CONFEDERATION (ITUC). $2^{\text {nd }}$ World Congress Resolution on extending social protection and ensuring good occupational health and 
Non sono peraltro indenni dai rischi per la propria salute e sicurezza nemmeno coloro che svolgono la propria attività in luoghi di lavoro che possono essere considerati modelli di democrazia e partecipazione nelle economie più sviluppate ${ }^{5}$ ed è ormai innegabile che il processo di globalizzazione genera conseguenze sulle condizioni di lavoro a livello mondiale, nelle economie sviluppate come in quelle emergenti o in via di sviluppo, sia sul piano delle situazioni individuali, sia su un più ampio livello di organizzazione del lavoro. ${ }^{6}$

\section{Il diritto internazionale dei diritti umani e la salute e sicurezza dei lavoratori: \\ La Dichiarazione Universale dei Diritti dell'Uomo e il Patto Internazionale sui diritti economici, sociali e culturali}

L'analisi del diritto alla salute e sicurezza dei lavoratori in una prospettiva di diritti umani, come già accennato, permette di attribuire ad esso caratteristiche di universalità ed inviolabilità non rintracciabili altrimenti.

In un'ottica di comparazione tra ordinamenti, osserviamo che la prospettiva della salute e sicurezza come diritto umano è stata adottata da alcuni autori come "nuova frontiera per le relazioni industriali" nel contesto statunitense ${ }^{7}$, come prospettiva teorica finalizzata a garantire una maggior tutela per i lavoratori più vulnerabili

safety. 2CO/E/6.12 (final). Vancouver: 2010. p. 3

5 ISHIDA, M. Death and suicide from overwork: the Japanese workplace and labour law. in CONAGHAN, J. et al. (a cura di). Labour law in an era of globalization: Transformative practices \& possibilities. Oxford: Oxford University Press, 2002. p. 219

6 MANZEY, D., MAROLD, J. Editorial. Occupational accidents and safety: The challenge of globalization. Safety Science, v. 37, 2009. p. 723. Cf per una trattazione più approfondita: WILPERT, B. Impact of globalization on human work. Safety Science, v. 37, 2009, p. 727-32. HÄMÄLÄINEN, P. The effect of globalization on occupational accidents. Safety Science, v. 37, 2009, p. 733-42. BARAM, M. Globalization and workplace hazards in developing nations. Safety Science, v. 37, 2009, p. 756-66.

7 HILGERT, J. A new frontier for industrial relations: workplace health and safety as a human right in GROSS J. A., COMPA, L. (a cura di) Human rights in labor and employment relations: international and domestic perspectives. Champaign, Ill: Labor and employment relations association, 2009. p. 43-71. L'autore sostiene che "la tutela della salute e sicurezza dei lavoratori è esemplificativa del contrasto tra l'economia del lavoro di stampo istituzionalista e i diritti umani” (p. 53). Per una tesi a sostegno della conciliabilità tra le due visioni si veda RANDALL WRAY, L., FORSTATER, M. Full employment and social justice in (a cura di) CHAMPLIN DELL P., KNOEDLER JANET T., The institutionalist tradition in Labor Economics. M.E. Sharpe Inc. 2004. Ringrazio il Prof. Jack Reardon, della Hamline University School of Business, per la disponibilità a confrontarsi sul tema e l'indicazione bibliografica in ultimo citata. 
e basata sull'assunto che "la salute e la sicurezza dei lavoratori sono diritti umani fondamentali, diritti economici e sociali che oggi rappresentano una delle più grandi crisi a livello globale" 8 .

La Dichiarazione Universale dei Diritti dell'Uomo, adottata dall'Assemblea Generale dell'Organizzazione delle Nazioni Unite (ONU) nel dicembre del 1948, afferma all'art. 23 c. 1 che "ogni individuo ha diritto al lavoro, alla libera scelta dell'impiego, a giuste e soddisfacenti condizioni di lavoro ed alla protezione contro la disoccupazione".

In modo più generale ma, a nostro parere, non meno incisivo, il precedente art. 22 afferma che

ogni individuo, in quanto membro della società, ha diritto alla sicurezza sociale, nonché alla realizzazione attraverso lo sforzo nazionale e la cooperazione internazionale ed in rapporto con l'organizzazione e le risorse di ogni Stato, dei diritti economici, sociali e culturali indispensabili alla sua dignità ed al libero sviluppo della sua personalità

E' poi all'interno del successivo Patto internazionale sui diritti economici, sociali e culturali, entrato in vigore nel 1976, che si rinviene un riferimento esplicito alla salute e sicurezza, la cui giustificazione etica è riconducibile, fin dal Preambolo del Patto, nella tutela della "dignità inerente alla persona umana"'.

Il Patto afferma, all'art. 7, il dovere degli Stati Parti di riconoscere "il diritto di ogni individuo di godere di giuste e favorevoli condizioni di lavoro, le quali garantiscano in particolare: (lett. b) la sicurezza e l'igiene del lavoro".

Il riconoscimento di questo diritto come corollario essenziale del diritto al lavoro sancito dall'art. 6 del Patto e dal citato art. 23 della Dichiarazione Universale, è dunque alla base del dialogo tra i due strumenti e della prospettiva teorica attraverso la quale stiamo analizzando la questione della tutela della salute e sicurezza ${ }^{10}$.

All'interno dello stesso Patto, inoltre, è inevitabile richiamare la stretta relazione con un altro diritto umano fondamentale quale il diritto alla salute, al quale si riferisce

8 HILGERT, J. A new frontier for industrial relations: workplace health and safety as a human right in GROSS J. A., COMPA L. (a cura di) Human rights in labor and employment relations: international and domestic perspectives. Champaign, Ill: Labor and employment relations association, 2009 , p. 68

9 CRAVEN, M. C. R. The International Covenant on Economic Social and Cultural Rights: a perspective on its development. Oxford: Clarendon, 1995. p. 22 e p. 226-247 per una trattazione più estesa del diritto in questione. Sul Patto in generale si veda SEPULVEDA, M.M. The nature of the obligations under the International Covenant on Economic, Social and Cultural rights. Antwerpen (etc.): Intersentia, 2003.

10 CRAVEN, M. C. R. The International Covenant on Economic Social and Cultural Rights: a perspective on its development. Oxford: Clarendon, 1995, p. 226. 
l'art. $12^{11} \mathrm{e}$ che deve essere inteso, secondo la definizione adottata dall'Organizzazione Mondiale della Sanità (OMS), come "uno stato di completo benessere fisico, mentale e sociale e non solamente l'assenza di malattia o infermità"

Il 10 dicembre del 2008, l'Assemblea Generale delle Nazioni Unite ha inoltre adottato per consenso ${ }^{13}$ il Protocollo Opzionale al Patto sui Diritti Economici, Sociali e Culturali, dove, all'art. 2, è prevista la possibilità di presentare comunicazioni da parte di individui o gruppi che lamentino la violazione di diritti tutelati dal Patto ${ }^{14}$. Il Protocollo non è tuttavia ancora entrato in vigore, non avendo ottenuto il numero

11 Rilevano, in particolare, la lett. b (miglioramento di tutti gli aspetti delligiene ambientale e industriale) e la lett. c (profilassi, cura e controllo delle malattie anche professionali). Si veda anche TOEBES, B. C. A. The right to health as a human right in international law. Antwerpen (etc.): Intersentia, 1999. p. 271 e DUPUY, R. J. (a cura di) The right to health as a human right. Alpen aan den Rijn: Sijthoff \& Noordhhoff, 1979. Il testo include (p. 227-31) un intervento di E. Hellen, all'epoca Direttore del Dipartimento per la sicurezza e la salute del lavoro dell'Ufficio Internazionale del Lavoro, che affronta il tema degli incidenti sul lavoro e delle malattie professionali. A conferma di tale interpretazione si veda anche COMITATO ONU PER I DIRITTI ECONOMICI SOCIALI E CULTURALI. General Comment n. 14 (2000). E/C.12/2000/4. 11 agosto 2000. par. 15 e 16 e LEVY FEITSHANS I. Occupational health as a human right. Reperibile su http://www.ilo.org/safework_bookshelf/english?content\&nd=857170259 (ultimo accesso 23 settembre 2010).

12 Le tendenze più recenti all'interno dell'OIL e dell'OMS vedono un'attenzione crescente al tema del benessere dei lavoratori. Il programma dell'OIL relativo a salute e sicurezza sul lavoro e ambiente (SafeWork) assume infatti la promozione della salute e del benessere sul luogo di lavoro come attività complementare rispetto alle misure di tutela della salute e sicurezza ed attuata tramite l'adozione di programmi di prevenzione ed assistenza nelle aree dell'abuso di droga ed alcol, dell'HIV/AIDS, dello stress lavoro-correlato, della violenza sul lavoro e della promozione di luoghi di lavoro liberi dal fumo. L'assunto è infatti che il benessere sul lavoro riguardi tutti gli aspetti della vita lavorativa, a partire dalla qualità e sicurezza dell’ambiente fisico di lavoro fino a considerare, in una prospettiva più ampia, l'influenza del clima e dell'organizzazione del lavoro. Si veda anche (a cura di) BAUMANN, A., et al. Mental health and well-being at the workplace: Protection and inclusion in challenging times. World Health Organization. 2010. Reperibile presso http://www.euro.who.int/_data/assets/pdf_ dile/0018/124047/e94345.pdf (ultimo accesso 11 novembre 2010). Tali tendenze sono state recepite anche nella legislazione italiana (Decreto Legislativo 9 aprile 2008, n. 81. Attuazione dell'articolo 1 della legge 3 agosto 2007, n. 123, in materia di tutela della salute e della sicurezza nei luoghi di lavoro). Esemplificativo è l'art. 28, che include nell'oggetto della valutazione dei rischi lo stress lavoro-correlato. Si vedano anche: CORBIZZI FATTORI, G., SIMONINI, F. Il benessere sul lavoro attraverso la valutazione dello stress lavoro-correlato: un convegno. ISL - Igiene e Sicurezza del Lavoro. n. 5 2010. p. 261 - 63; DAMIANI, L. INAIL e benessere organizzativo: nuove prospettive di tutela per i dipendenti. I diritti dell'uomo. n. 2 2008. p. 53 - 57; GALLO CARRABBA, A. La progettazione del benessere nei luoghi di lavoro dal d. lgs. 626/94 ai modelli di wellness management. L'Amministrazione italiana. n. 4 2008. p. 487 - 507; MALZANI, F. Obbligo di sicurezza e nuove latitudini: lo stress da lavoro (Nota a Cassazione sezione lavoro 7 giugno 2007, n. 13309). La Nuova Giurisprudenza Civile Commentata. n. 12008 . p. $34-39$.

13 Risoluzione A/RES/63/117

14 Il successivo art. 3 stabilisce le condizioni di ammissibilità delle comunicazioni, tra le quali è previsto l'esaurimento dei ricorsi interni previsti dall'ordinamento statale. 
minimo di 10 ratifiche ${ }^{15}$.

L'adozione di una prospettiva basata sui diritti umani è peraltro auspicata anche all'interno dell'Unione Europea, se si considera che il Parlamento Europeo, nella Risoluzione del maggio 2007 relativa alla garanzia di un lavoro dignitoso per tutti ${ }^{16}$ "invita la Commissione, gli Stati Membri e le Parti Sociali a riconoscere che standard elevati in materia di salute e sicurezza sul luogo di lavoro costituiscono un diritto umano essenziale".

\section{L'Organizzazione Internazionale del Lavoro: il ruolo della salute e sicurezza nelle politiche più recenti}

Il diritto alla salute e sicurezza sul lavoro, oltre ad essere affermato dai più importanti strumenti internazionali relativi ai diritti umani, come appena evidenziato, è centrale anche rispetto agli strumenti ed alle politiche dell'Organizzazione Internazionale del Lavoro (OIL).

La Dichiarazione di Filadelfia, riguardante gli scopi e gli obiettivi dell'Organizzazione e risalente al maggio 1944, afferma al par. 3 “il solenne impegno da parte dell'OIL di assecondare la messa in opera, nei vari Paesi del mondo, di programmi atti a realizzare" (lett. g) "una protezione adeguata della vita e della salute dei lavoratori, qualunque sia la loro occupazione".

In base alle tendenze più recenti, l'attività dell'OIL è orientata, da una parte, all'analisi ed alla prevenzione dei rischi emergenti come conseguenza dei mutamenti nel mondo del lavoro e delle più ampie tendenze generate dalla globalizzazione e, dall'altra, a raggiungere un numero di ratifiche ed una attuazione più efficace degli strumenti di portata internazionale relativi alla salute e sicurezza ${ }^{17}$, identificati nella Convenzione n. 155 del $1981^{18}$, nel Protocollo Addizionale del $2002^{19}$ e nella

15 Gli Stati firmatari sono 33, mentre le ratifiche finora (settembre 2010) pervenute sono solo quelle di Ecuador e Mongolia.

16 PARLAMENTO EUROPEO. Risoluzione del 23 maggio 2007 sulla promozione di un lavoro dignitoso per tutti (2006/2240(INI)) in Gazzetta Ufficiale dell'Unione Europea C 102 E/321 del 24 aprile 2008.

17 ORGANIZZAZIONE INTERNAZIONALE DEL LAVORO. Plan of action (2010-2016) to achieve widespread ratification and effective implementation of the occupational safety and health instruments (Convention No. 155, its 2002 Protocol and Convention No. 187). Adottato dal Comitato Esecutivo dell'OIL nel corso della sua $307 \mathrm{ma}$ sessione, nel marzo 2010. Reperibile presso http://www.ilo.org/wcmsp5/groups/public/---ed_norm/---normes/ documents/genericdocument/wcms_125616.pdf. Ultimo accesso 9 novembre 2010.

18 Occupational Safety and Health Convention, 1981 (No. 155) ratificata da 56 Stati (Fonte: ILOLEX 5.11.2010)

19 Protocol of 2002 to the Occupational Safety and Health Convention ratificato da 7 Stati (Fonte: 
Convenzione n. 187 del $2006^{20}$.

Il Piano d'Azione prevede, oltre al suddetto obiettivo, più strettamente giuridico, di promozione delle ratifiche, una serie di altri obiettivi strategici, tra cui lo sviluppo della cultura della prevenzione ed il miglioramento delle condizioni di salute e sicurezza nelle Piccole e Medie Imprese (PMI) e nell'economia informale. Di non secondaria importanza, inoltre, è l'obiettivo di migliorare la raccolta, l'analisi e la diffusione di informazioni statistiche riguardanti salute e sicurezza dei lavoratori, attraverso azioni promozionali, assistenza tecnica e l'elaborazione di metodologie e di indicatori adeguati.

Vale la pena inoltre accennare, seppur brevemente, ai contenuti dei tre strumenti sulla cui promozione l'OIL investirà da qui fino al 2016.

La Convenzione n. 155 del 1981 concerne l'elaborazione, l'attuazione e la revisione periodica di una politica nazionale relativa alla tutela della salute e sicurezza dei lavoratori e dell'ambiente di lavoro ${ }^{21}$, attraverso la consultazione delle organizzazioni dei datori di lavoro e dei lavoratori considerate più rappresentative. Per quanto concerne la singola impresa ${ }^{22}$, ricade sui datori di lavoro l'obbligo di garantire condizioni di lavoro sane e sicure.

Il Protocollo Addizionale del 2002 richiede l'elaborazione di un sistema per la registrazione e la notifica di incidenti sul lavoro, malattie professionali (anche in casi di mero sospetto) e eventi pericolosi, nonché la pubblicazione di statistiche annuali a livello nazionale relative a questi fenomeni.

La più recente Convenzione n. 187 del 2006 riguarda invece lo sviluppo, in consultazione con le organizzazioni maggiormente rappresentative dei datori e dei lavoratori, di politiche, sistemi e programmi nazionali per il miglioramento continuo delle condizioni di salute e sicurezza.

Nessuno di questi strumenti è stato, peraltro, ratificato dall'Italia ${ }^{23}$.

Un ulteriore passo rilevante è stato compiuto nel 2009 in occasione della Giornata Mondiale dedicata al tema della salute e sicurezza dei lavoratori ${ }^{24}$. In questa occasione

\section{ILOLEX 5.11.2010)}

20 Promotional Framework for Occupational Safety and Health Convention, 2006 (No. 187) ratificata da 16 Stati (Fonte: ILOLEX 5.11.2010)

21 Art. 4

22 Art. 16

23 Cf SENAto Della RePubbliCA, XVI Legislatura. Commissione Parlamentare di Inchiesta sul fenomeno degli infortuni sul lavoro con particolare riguardo alle cosiddette "morti bianche". Audizione di Rappresentanti dell'OIL. 42a seduta, martedì 26 gennaio 2010. Testo reperibile su http?//www.senato.it/commissioni/156058/156060/156062/277175/278191/ schedasedutacommissione.htm_(ultimo accesso 23 settembre 2010).

24 ORGANIZZAZIONE INTERNAZIONALE DEL LAVORO. Health and life at work: a basic human right. 2009. Reperibile su http://www.ilo.org/public/portuge/region/europro/ 
infatti è stato affermato che la protezione dalle malattie e dagli infortuni derivanti dallo svolgimento della propria attività lavorativa non costituisce solamente un diritto proprio del lavoratore, ma un diritto umano fondamentale la cui tutela è uno degli obiettivi principali dell'Organizzazione, che si trova a dover affrontare le complicazioni derivanti da cambiamenti organizzativi e da processi di ristrutturazione aziendale quali il maggior ricorso a esternalizzazioni (outsourcing), all'impiego di lavoratori part-time ed al sub-appalto ${ }^{25}$, nonché dall'attuale crisi economica e sociale.

La prospettiva appena delineata si pone peraltro in una linea di continuità e coerenza rispetto alla precedente Decent Work Agenda, elaborata dall'OIL a partire dal $1999^{26}$ e con la quale non hanno potuto fare a meno di confrontarsi le istituzioni dell'Unione Europea, come dimostra anche la risoluzione adottata dal Parlamento Europeo nel 2007 sulla promozione di un lavoro dignitoso per tutti ${ }^{27}$.

Il quadro finora delineato può dunque far ritenere superabili alcune critiche rivolte alla riduzione, da parte della stessa OIL, dei cosiddetti core labour rights a quattro diritti fondamentali. In particolare, facciamo qui riferimento alla Dichiarazione sui principi e i diritti fondamentali nel lavoro del $1998^{28}$, dove veniva sancito l'obbligo per tutti i Membri dell'Organizzazione, in quanto tali e quindi a prescindere dalla ratifica delle relative convenzioni, di rispettare, promuovere e realizzare i principi connessi ai seguenti diritti fondamentali: libertà di associazione e riconoscimento effettivo del diritto di contrattazione collettiva; eliminazione di ogni forma di lavoro forzato o obbligatorio; abolizione effettiva del lavoro infantile; eliminazione della discriminazione in materia di impiego e professione ${ }^{29}$.

L'assenza di riferimenti alla tutela della salute e sicurezza dei lavoratori aveva dunque portato alcuni autori a criticare la riduzione della relazione tra lavoro e diritti umani ai quattro suddetti diritti ${ }^{30}$. Il concetto di "lavoro decente" comprende,

lisbon/pdf/28abril_09_en.pdf. Ultimo accesso 9 novembre 2010.

25 ORGANIZZAZIONE INTERNAZIONALE DEL LAVORO. Health and life at work: a basic human right. 2009. p. 9

26 MAUPAIN, F. New foundation or new façade? The ILO and the 2008 Declaration on Social Justice for a fair globalization. The European Journal of International Law. vol. 20 n. 3. 2009. p. 837-38. L'autore sottolinea la definizione sintetica e condivisa del concetto di decent work, nonché la sua "malleabilità".

27 PARLAMENTO EUROPEO. Risoluzione del 23 maggio 2007 sulla promozione di un lavoro dignitoso per tutti (2006/2240(INI) in Gazzetta Ufficiale dell'Unione Europea C 102 E/321 del 24 aprile 2008.

28 Testo completo reperibile su http://www.ilo.org/publi/italian/region/eurpro/rome/standaeds/dirfond.htm. Cf in particolare par. 2

29 Cf anche HEPPLE, B. Labour laws and global trade. Oxford; Portland, Or.: Hart Publishing. 2005. p. 57.

30 MUNDLAK, J. The right to work - The value of work in (a cura di) BARAK-EREZ D., GROSS A.M. Exploring social rights: Between theory and practice. Oxford; Portland, 
infatti, la garanzia della salute e della sicurezza per tutti i lavoratori, compresi coloro che svolgono le proprie attività al di fuori dell'economia formale ${ }^{31}$.

Certo, resta il problema dell'incompletezza dei dati raccolti, che si limitano spesso a rappresentare l'economia formale, limitando le possibilità di analisi e di intervento in settori che, soprattutto nei Paesi in via di sviluppo, costituiscono le aree allo stesso tempo più critiche e più diffuse, quali, ad esempio, il lavoro nelle aziende agricole, nelle aree maggiormente degradate o il lavoro domestico delle donne ${ }^{32}$.

\section{Il Consiglio d'Europa e i diritti economici e sociali: la Carta Sociale Europea}

La Carta sociale europea riveduta, aperta alla firma degli Stati membri del Consiglio d'Europa, a Strasburgo, il 3 maggio 1996, ed entrata in vigore il $1^{\circ}$ luglio 1999, rappresenta uno degli strumenti più avanzati dal punto di vista della tutela della salute e della sicurezza sul lavoro come diritto umano, se non, più in generale, come strumento del Consiglio d'Europa dedicato in modo specifico alla tutela dei diritti economici e sociali ${ }^{33}$. Essa, fin dalla versione originaria del 1961, può essere ritenuta il primo strumento internazionale a sostegno della tutela del diritto a condizioni di lavoro sane e sicure in quanto diritto umano ${ }^{34}$.

Il Comitato Europeo dei Diritti Sociali, composto da quindici esperti indipendenti, è l'organo incaricato di verificare il rispetto della Carta da parte degli Stati che l'hanno ratificata ${ }^{35}$.

Oregon: Hart. 2007. p. 348

31 Sono considerati tali, ad esempio, i lavoratori domestici e quelli autonomi. Cf GHAI, D. Decent work: Concept and indicators. International labour review. v. 142 n. 2 2003. p. 113, 1212003

32 GHAI, D. Decent work: Concept and indicators. International labour review. v. 142 n. 2 2003. p. 122

33 La Carta Sociale è un trattato internazionale che, a seguito del processo di revisione rispetto alliniziale versione del 1961, ha incluso una serie di nuovi diritti, tra i quali citiamo: il diritto alla protezione contro molestie sessuali ed altre forme di molestie; diritti dei lavoratori aventi responsabilità familiari all'uguaglianza di opportunità e di trattamento; diritti dei rappresentanti dei lavoratori. Essa ha inoltre apportato delle modifiche a favore del rafforzamento del principio di non discriminazione, del miglioramento dell'eguaglianza tra donne e uomini, della maggiore protezione della maternità, delle madri, dei minori lavoratori e dei disabili. L'applicazione della nuova Carta è sottoposta allo stesso dispositivo di controllo previsto dalla Carta del 1961, rafforzato in seguito dal Protocollo del 1991 e dal Protocollo del 1995, che prevede un sistema di reclamo collettivo attivabile da organizzazioni sindacali e datoriali e organizzazioni non governative (ONG).

34 LASAK, K. Health and safety in the European Social Charter. International Journal of Comparative Labour Law and Industrial Relations. v. 25 n. 2 2009. p. 111

35 Il Comitato ha il compito di esaminare i rapporti inviati annualmente dagli Stati Parti, riguardo i quali 
Quali sono, dunque, le disposizioni rilevanti previste nella versione riveduta della Carta entrata in vigore nel $1999^{36}$ ?

Innanzitutto, l'art. 2, che stabilisce il diritto ad eque condizioni di lavoro, al par. 4 prevede l'impegno delle Parti ad eliminare i rischi inerenti ai lavori pericolosi o insalubri e, nel caso in cui tali rischi non possano essere eliminati o sufficientemente ridotti, a garantire ai lavoratori impiegati in tali occupazioni sia una riduzione della durata del lavoro, sia ferie retribuite supplementari.

Il successivo art. 3 concerne nello specifico il diritto alla sicurezza e all'igiene sul lavoro, prevedendo l'impegno delle Parti a: (par. 1) definire, attuare e riesaminare periodicamente una politica nazionale coerente in materia di sicurezza e salute dei lavoratori e dell'ambiente di lavoro; (par. 2) promulgare regolamenti relativi alla sicurezza e all'igiene e (par. 3) prevedere misure di controllo della loro applicazione; (par. 4) promuovere l'istituzione progressiva sul lavoro di servizi sanitari con funzioni sostanzialmente preventive e di consulenza per tutti i lavoratori.

L'art. 11 include la prevenzione degli infortuni tra gli obiettivi delle misure volte ad assicurare effettività al diritto alla protezione della salute.

Il diritto dei lavoratori e dei loro rappresentanti a partecipare alla determinazione ed al miglioramento delle condizioni e dell'ambiente di lavoro è sancito dall'art. 22, mentre l'art. 26, recante l'intestazione "diritto alla dignità del lavoro", ha l'obiettivo di promuovere la pubblicizzazione, l'informazione e la prevenzione, nonché l'adozione di misure adeguate a tutela dei lavoratori, in materia di molestie sessuali e atti condannabili o esplicitamente ostili o offensivi posti in essere sul luogo di lavoro o in connessione con il lavoro.

Dal 2006 è stato adottato un sistema di rapporti statali tematici, il cui secondo round, dedicato a "Salute, sicurezza sociale e protezione sociale", ha riguardato anche gli articoli 3 e 11 della Carta Sociale.

Nel Rapporto dedicato alle attività del $2009^{37}$ il Comitato rileva il permanere di problematiche connesse alla salute e sicurezza sul lavoro. I problemi di conformità maggiormente rilevanti sono stati identificati nella restrizione dell'ambito di applicazione soggettivo della legislazione sulla protezione dei lavoratori, nell'assenza di adeguati

adotta delle conclusioni, e i ricorsi collettivi presentati, che vengono giudicati tramite decisioni.

36 Per l'interpretazione dei singoli articoli elaborata dal Comitato si veda COMITATO EUROPEO DEI DIRITTI SOCIALI Digest of the Case Law of the European Committee of Social Rights, settembre 2008. Reperibile su http://www.coe.int/t/gdh/monitoring/socialcharter/ Digest/DigestIndex_en.asp. Ultimo accesso 9 novembre 2009.

37 COMITATO EUROPEO DEI DIRITTI SOCIALI. Activity Report 2009. 25 giugno 2010. Reperibile su http://www.coe.int/t/gdh/monitoring/socialcharter/Presentation/ ActivityReport2009_en.pdf. Ultimo accesso 9 novembre 2010. 
meccanismi ispettivi e di strategie di prevenzione, nonché, in alcuni casi, di insufficienti informazioni statistiche relative a infortuni e malattie occupazionali. E' importante sottolineare, soprattutto in un'ottica di Responsabilità Sociale d'Impresa, che le suddette lacune permangono anche in un contesto di formale rispetto, a livello istituzionale, delle norme, dove però è la pratica a non riflettere la prescrizione giuridica.

Per quanto concerne la presentazione di ricorsi collettivi da parte di organizzazioni sindacali e di Organizzazioni Non Governative nazionali ed internazionali, possiamo citare in quanto pertinente ai diritti oggetto della trattazione il caso Marangopoulos Foundation for Human Rights (MFHR) c. Grecia $^{38}$, dove veniva lamentata la violazione degli art. 2 par. 4, art. 3 par. 1 e 2 e art. 11 della Carta Sociale Europea. Nella fattispecie, veniva fatto riferimento agli effetti negativi dell'inquinamento ambientale sulla salute dei lavoratori e degli abitanti in un'area di estrazione e lavorazione della lignite da parte di un'azienda greca di cui lo Stato era il maggior azionista e su cui esercitava un controllo diretto.

Il Comitato dei Ministri del Consiglio d'Europa, nella risoluzione finale adottata nel gennaio 2008, ha constatato la violazione da parte del Governo greco di una serie di obblighi: informazione delle persone residenti nelle aree di estrazione della lignite; monitoraggio del rischio epidemiologico; attuazione dei regolamenti concernenti la salute e la sicurezza sul lavoro ${ }^{39}$.

\section{Quali spunti di riflessione nel diritto del lavoro italiano?}

L'analisi svolta in seguito dei contributi di alcuni tra $i$ principali giuslavoristi italiani, senza pretesa di esaustività, ha lo scopo di evidenziare alcune idee chiave alla base di sviluppi del diritto del lavoro già in essere o prospettati, che possano rendere quest'area del diritto, tradizionalmente legata ai confini nazionali, maggiormente aperta ed adattabile alle sfide poste dall'internazionalizzazione dell'economia.

In primo luogo, la tutela della salute e della sicurezza dei lavoratori può essere collocata a nostro parere nel più ampio spettro delle questioni attinenti la "dimensione transnazionale del lavoro nell'economia globale ${ }^{40 "}$. Non si può fare

38 Ricorso n. 30/2005

39 Il caso è citato anche in FIDH - International Federation for Human Rights. Corporate Accountability for Human Rights Abuses: A guide for victims and NGOs on recourse mechanisms. 2010. Reperibile presso http://fidh.org/CoporateAccountability-for-HumanRights-Abuses .Ultimo accesso 9 novembre 2010. Cf p. 114-15

40 L'espressione è tratta dal paper "Verso un nuovo lessico giuslavoristico: inventario di questioni", redatto dal Prof. Marcello Pedrazzoli, docente di Diritto del Lavoro presso l'Università di Bologna, che ringrazio per averlo reso disponibile in occasione del seminario svolto il 2 luglio 
a meno, infatti, di ritenere che la tutela della salute e della sicurezza in quanto diritto umano, e quindi su scala universale, metta in discussione i tradizionali limiti territoriali del diritto del lavoro ${ }^{41}$. Alla delocalizzazione dei processi produttivi spesso i più rischiosi proprio per la salute e la sicurezza dei lavoratori - verso Paesi dove l'efficacia e l'effettività delle tutele sono inferiori, si aggiungono gli effetti determinati dai movimenti migratori, che spesso comportano l'impiego di soggetti più vulnerabili e meno tutelati in settori ed occupazioni con maggiori livelli di rischio, se non del tutto appartenenti all'ambito dell'economia sommersa e del lavoro illegale ${ }^{42}$.

L'abbassamento delle tutele a fini di dumping sociale e l'ipotesi che per le economie dei Paesi emergenti ne derivino vantaggi competitivi e quindi la possibilità di crescita economica, se posti a confronto con l'idea che salute e sicurezza necessitano di una tutela rafforzata in quanto diritti umani, portano ad interrogarsi sui costi umani ed ambientali della crescita economica e della competizione internazionale e a chiedersi quale sia l'optimum non solo nell'utilizzo dei fattori produttivi, ma anche degli strumenti regolativi. In questa direzione svolge a nostro parere un ruolo fondamentale l'attività dell'OIL, volta, come visto in precedenza, a fissare standard minimi, o, se si preferiscono altre aggettivazioni, essenziali ed inderogabili a tutela della dignità dell'uomo ${ }^{43}$.

2010 presso la Fondazione Marco Biagi dell’Università di Modena e Reggio Emilia.

${ }^{41}$ Per riferimenti alla più recente normativa italiana in materia di salute e sicurezza (Decreto legislativo 9 aprile 2008 n. 81) si vedano ANTONUCCI, M. Il campo di applicazione oggettivo e soggettivo della nuova normativa in materia di sicurezza e tutela della salute dei lavoratori. Diritto delle Relazioni Industriali n. 22008 p. 441-60 e PASCUCCI, P. Il campo di applicazione soggettivo delle nuove regole sulla salute e sicurezza nei luoghi di lavoro. Diritti Lavori Mercati n. 22008 p. 297-338, nonché LASSANDARI, A. La sicurezza del lavoro in (a cura di) VALLEBONA, A. I contratti di lavoro. Tomo I. Torino: UTET giuridica; Milanofiori, Assago: Wolters Kluwer Italia, 2009, p. 641-80.

Sul legame tra disciplina del lavoro, sovranità nazionale e competitività al ribasso si veda anche l'autorevole contributo di uno dei massimi giuslavoristi spagnoli: RODRIGUEZ-PIÑERO Y BRAVO-FERRER, M. Il diritto del lavoro davanti alla sfida della globalizzazione in NAPOLI M. (a cura di) Globalizzazione e rapporti di lavoro. Milano: Vita e Pensiero, 2006. p. 121-22

42 Si veda anche PESSI, R. Lavoro, mercato, "ordine spontaneo", regolazione transnazionale. Rivista Italiana di Diritto del Lavoro n. 32009 p. 277-97. L'autore sottolinea, oltre al venir meno dell'effettività delle tecniche regolative a causa della delocalizzazione, anche un processo di "dematerializzazione"del lavoro, che dà origine a "identità cangianti estranee a un minimo denominatore comune" (ivi p. 281). Concetti analoghi sono espressi in PESSI, R. Persona, lavoro e poteri privati. Argomenti di diritto del lavoro n. 32009 p. 681

43 Cf anche RODRIGUEZ-PIÑERO Y BRAVO-FERRER, M. Il diritto del lavoro davanti alla sfida della globalizzazione in NAPOLI M. (a cura di) Globalizzazione e rapporti di lavoro. Milano: Vita e Pensiero, 2006. p. 126 e 131.

Su una relazione positiva tra rispetto dei diritti fondamentali del lavoro e competitività internazionale si veda anche RICCI, M. Ambiente di lavoro e Responsabilità Sociale d'Impresa in (a cura di) MONTUSCHI, L., TULLINI, P. Lavoro e Responsabilità Sociale d'Impresa. Bologna: Zanichelli, 2006, p. 37 
All'idea che le responsabilità del diritto del lavoro non possano più limitarsi alla garanzia dell'inderogabilità delle norme e della stabilità delle tutele all'interno dell'ordinamento statale o europeo, è necessario affiancare una riflessione sulle tecniche più efficaci affinché le tutele siano più estese possibili ed equamente distribuite anche tra i lavoratori coperti da minori garanzie e maggiormente esposti allo sfruttamento della propria manodopera, dove i rischi per la salute e la sicurezza sono tra i più immediati.

Di conseguenza, l'individuazione degli strumenti più efficaci per garantire il rispetto ed eventualmente sanzionare le violazioni del diritto alla salute e alla sicurezza apre, a nostro parere, una prospettiva più ampia, che sappia non solo riconoscere l'esistenza di diversi livelli di tutela - e le rispettive competenze - a livello nazionale, comunitario ed internazionale, ma che sappia anche coniugare gli strumenti offerti dalla hard law con quelli di soft law, senza assumere a priori che il monopolio dell'una o dell'altra sia la soluzione migliore, ma ritenendo che entrambe, se utilizzate correttamente, possano essere strumenti di giustizia sociale ${ }^{44}$.

A questo proposito, una questione certo non facile da dirimere, e per la quale non esiste molto probabilmente una soluzione universalmente valida, è quella relativa all'an ed al quantum della normativa imperativa ed inderogabile, posto che il pieno funzionamento di quest'ultima può essere garantito solo se copre l'intero mercato di riferimento, determinando, in caso contrario, la delocalizzazione delle attività economiche all'interno di mercati regolati da norme meno stringenti ${ }^{45}$.

A parere di chi scrive, la giusta commistione tra i due strumenti può variare, in un'ottica globale, a seconda dei contesti economici, sociali e culturali di riferimento $\mathrm{e}$, in secondo luogo, l'utilizzo di strumenti soft o volontaristici non deve escludere l'esistenza di norme che ne possano determinare contenuti e standard applicativi ${ }^{46}$.

In sintesi, riprendendo alcuni lemmi tra quelli individuati da Pedrazzoli, l'inclusione della tutela della salute e della sicurezza tra i diritti umani dei lavoratori può influire positivamente sulle dinamiche della competizione tra ordinamenti con l'obiettivo di mitigare la corsa al ribasso a cui è soggetta in generale la tutela dei diritti.

44 Premessa fondamentale, a questo riguardo, è che "il fine del diritto, e quindi anche del diritto del lavoro, è la realizzazione della giustizia sociale". Cf VALLEBONA, A. Fini e tecniche del diritto del lavoro in (a cura di) VALLEBONA, A. I contratti di lavoro. Tomo I. Torino: UTET giuridica; Milanofiori, Assago: Wolters Kluwer Italia, 2009, p. 3.

45 Cf VAlLEBOnA, A. "Caritas in Veritate" e diritto del lavoro. Rivista Italiana di Diritto del Lavoro n. 12009 p. 443

46 Si veda anche PERULLI, A. Corporate social responsibility e diritto del lavoro in (a cura di) PERULLI, A. L'impresa responsabile: Diritti sociali e Corporate Social Responsibility. Matelica: Halley, 2007, p. 109 
Connesso a questo primo aspetto potrebbe essere un crescente ruolo del contratto collettivo, sia a livello nazionale che transnazionale ${ }^{47}$, nonché una rivisitazione di quelli che finora, in parte della letteratura, sono stati definiti "diritti umani dei lavoratori" ${ }^{48}$.

\section{Quale ruolo per la Responsabilità Sociale d'Impresa?}

Prendendo spunto da quanto messo in luce finora, possiamo affermare che il ruolo attribuibile a pratiche di Responsabilità Sociale d'Impresa (RSI) è oggetto di crescente attenzione anche da parte di studiosi del diritto del lavoro.

Nel contesto italiano lo testimonia, ad esempio, il "Manifesto di Venezia per la regolazione della globalizzazione economica. La globalizzazione virtuosa” del $2009^{49}$, dove si afferma, ad esempio, "la necessità di un marchio sociale ed ambientale (...)

47 Cf PESSI, R. Persona, lavoro e poteri privati. Argomenti di diritto del lavoro n. 32009 p. 683, GALIZIA, C. Responsabilità sociale delle imprese ed autonomia collettiva. Diritti Lavori Mercati n. 12009 p. 191-219, SCARPONI, S. La "privatizzazione" delle norme internazionali di tutela del lavoro: codici etici di responsabilità sociale, accordi sindacali internazionali. Lavoro e Diritto n. 32009 p. 403-425, dove l'autrice sottolinea anche il ruolo svolto dai comitati aziendali europei (ivi p. 410).

Si veda anche il recente rapporto relativo alle clausole in materia di salute e sicurezza sul posto di lavoro e ambiente negli Accordi quadro internazionali, negoziati tra aziende multinazionali e Federazioni sindacali globali, elaborato dalla CONFEDERAZIONE EUROPEA DEI SINDACATI (CES-ETUC) Occupational health and safety and environmental clauses in International Framework Agreements: A study. Reperibile su http://www.etuc.org/ IMG/pdf/Global_Report-version_finale_anglaise-3pdf. Ultimo accesso 9 novembre 2010. Si vedano, in particolare, p. 27-31 e 49-60.

Gli Accordi Quadro Internazionali sono anche citati tra gli strumenti volontari di RSI dotati di maggior efficacia nell'accrescere l'accountability delle imprese in FIDH - International Federation for Human Rights. Corporate Accountability for Human Rights Abuses: A guide for victims and NGOs on recourse mechanisms, 2010. Reperibile presso http://www. fidh.org/Corporate-Acoountability-for-Human-Rights-Abuses. Ultimo accesso 9 novembre 2010. Cf p. 515-20.

48 Si vedano, a titolo esemplificativo, i già citati: HEPPLE, B. Labour laws and global trade. Oxford; Portland, Or.: Hart Publishing, 2005 e MUNDLAK, J. The right to work - The value of work in (a cura di) BARAK-EREZ D., GROSS A.M. Exploring social rights: Between theory and practice. Oxford; Portland, Oregon: Hart. 2007. Si veda inoltre ALSTON, P. (a cura di). Labour rights as human rights. Oxford: Oxford University Press. 2005 e HEPPLE, B. (a cura di). Social and labour rights in a global context: International and comparative perspectives. Cambridge: Cambridge University Press, 2002.

Per un sintetico riferimento alla violazione del diritto alla salute ed alla sicurezza nel contesto giuridico anglosassone si veda CLAPHAM, A. Human rights obligations of non-State actors. Oxford: Oxford University Press, 2006. p. 199-200.

49 PERULLI, A., MARRELLA, F. (estensori) Manifesto di Venezia per la regolazione della globalizzazione economica: La globalizzazione virtuosa. Rivista Italiana di Diritto del Lavoro n. 32009 p. $341-48$. 
sia in ragione della debolezza della disciplina imperativa multilaterale ed europea, sia in ragione dell'efficacia reale dei meccanismi di regolazione che utilizzano l'eco/ social labelling ${ }^{50 "}$ come nuovo strumento di garanzia dei diritti economici e sociali e la necessità di "promuovere l'effettività dell'autoregolazione dei poteri privati economici nella direzione di una cittadinanza sociale dell’impresa" ${ }^{51}$.

Da questo punto di vista è importante ricordare la pubblicazione, nel novembre 2010, del nuovo standard ISO 26000. Lo standard, elaborato dalla International Organization for Standardization (ISO), è costituito da una serie di linee guida ${ }^{52}$ per la responsabilità sociale nelle imprese private e nel settore pubblico ed è stato sviluppato, a partire dal 2005, all'interno di un gruppo di lavoro composto dai diversi stakeholders (rappresentanti di industria, Governi, lavoratori, consumatori, organizzazioni non governative), rappresentativo delle diverse aree geografiche e bilanciato in termini di rappresentanza di genere. Il testo dello standard ${ }^{33}$ include tra le linee guida il rispetto dei diritti umani riconosciuti a livello internazionale, a prescindere dai Paesi, dalle culture e dalle situazioni concrete in cui le imprese si trovano ad operare, e la necessità di evitare di trarre vantaggi da situazioni in cui i diritti umani non godono di appropriata protezione. La protezione della salute e della sicurezza sul lavoro è inclusa nella più ampia garanzia di condizioni di lavoro dignitose.

Il legame tra RSI e sicurezza e salute sul lavoro è stato colto anche dall'Agenzia Europea per la Sicurezza e la Salute sul Lavoro ${ }^{54}$, che ha raccolto ed analizzato vari tipi di iniziative internazionali, sia a livello europeo che globale, aventi come comune denominatore l'inclusione di strumenti volontari per una migliore tutela delle condizioni e dell'ambiente di lavoro.

50 PERUlLi, A., MARRELLA, F. (estensori) Manifesto di Venezia per la regolazione della globalizzazione economica: La globalizzazione virtuosa. Rivista Italiana di Diritto del Lavoro n. 32009 p. 345

51 PERULLI, A., MARRELLA, F. (estensori) Manifesto di Venezia per la regolazione della globalizzazione economica: La globalizzazione virtuosa. Rivista Italiana di Diritto del Lavoro n. 32009 p. 345-46. Tra le proposte effettuate evidenziamo il riferimento alla promozione di accordi sindacali transnazionali e la diffusione delle best practices europee sulla negoziazione collettiva dei codici di condotta.

52 Non utilizzabili, quindi, a fini di certificazione.

53 La versione considerata, data la recentissima approvazione della versione definitiva, è l'ultima bozza fatta circolare per le consultazioni a livello internazionale (Draft International Standard ISO/DIS 26000: Guidance on social responsibility).

${ }^{54}$ AGENZIA EUROPEA PER LA SALUTE E LA SICUREZZA SUL LAVORO. Responsabilità Sociale delle Imprese e Sicurezza e Salute sul Lavoro. Lussemburgo: Ufficio delle pubblicazioni ufficiali delle Comunità Europee, 2005. Si veda anche MONTERO, M. J. et al. Occupational Health and Safety in the framework of corporate social responsibility. Safety Science vol. 472009 p. 1440-45 per un'analisi dei contenuti dei principali strumenti di gestione della RSI a livello internazionale. 
Un aspetto certo rilevante per il tema in oggetto è inoltre quello relativo al trasferimento, a livello internazionale, dei rischi connessi alla produzione ${ }^{55}$, dal momento che politiche di RSI possono compensare gli insufficienti trasferimenti delle competenze per gestire le tecnologie esportate e innescare meccanismi di controllo da parte di diversi attori, quali gli acquirenti di prodotti provenienti da imprese e settori industriali particolarmente esposti a rischi per la salute e la sicurezza dei lavoratori.

\section{Osservazioni conclusive}

Lo scopo di questo articolo, come affermato nell'introduzione, è quello di capire se e con che modalità è possibile instaurare relazioni positive tra diversi ambiti di riflessione, con il fine di individuare nuove soluzioni per garantire una tutela il più estesa ed efficace possibile di un diritto inviolabile quale quello alla salute ed alla sicurezza dei lavoratori.

Quello che si è cercato di fare, innanzitutto, è stato individuare alcune delle principali e più recenti tendenze di sviluppo del tema a livello internazionale - ONU, OIL, Consiglio d'Europa - comunitario e, anche se limitatamente ad un approccio al diritto più teorico e meno attento alle prescrizioni normative, nazionale. Ciò è avvenuto sulla scorta della consapevolezza che, nel dibattito inerente la validità dei diversi strumenti di RSI, è necessario avere ben chiaro, in primo luogo, qual è il livello di tutela raggiunto dalle prescrizioni normative e quali le diverse modalità esperibili certo con diversi gradi di efficacia - nell'ottica di una tutela multilivello dei diritti che sappia allo stesso tempo adattarsi a contesti spesso molto diversi tra loro.

L'ipotesi qui sostenuta è che, in una prospettiva di analisi della validità e dell'efficacia degli strumenti di RSI, non si perda di vista il contesto giuridico nel quale tali pratiche si collocano ${ }^{56}$, ma anzi si tengano in considerazione le potenzialità di tali strumenti nell'espandere le frontiere della tutela effettiva dei diritti umani. Questo presuppone una non scontata ma a nostro parere positiva contaminazione tra ambiti disciplinari differenti, sia all'interno del diritto che in relazione ad altre

55 OLSEN O. E., LINDØE P. H., Risk on the ramble: The international transfer of risk and vulnerability. Safety Science vol. 472009 p. 746

56 Essendo la RSI un concetto derivato dal mondo anglosassone e da ordinamenti di common law, sarebbe auspicabile, anche se al di fuori della portata del presente articolo, analizzare quali modalità esistono, in ordinamenti differenti dal nostro, per la tutela, in generale, dei diritti dei lavoratori in ambito extraterritoriale, affinché un'analisi di stampo comparativo possa individuare elementi potenzialmente innovativi per il nostro ordinamento e sappia verificarne modalità e limiti della trasposizione. 
discipline, quali ad esempio la sociologia e l'economia, e la collaborazione tra gli attori impegnati a vario titolo e a diversi livelli per la promozione della RSI e la garanzia dei diritti dei lavoratori (sindacati, ONG, enti di certificazione, imprese e organizzazioni datoriali, istituzioni pubbliche). 


\section{Bibliografia}

AGENZIA EUROPEA PER LA SICUREZZA E LA SALUTE SUL LAVORO. Responsabilità Sociale delle Imprese e Sicurezza e Salute sul Lavoro. Lussemburgo: Ufficio delle pubblicazioni ufficiali delle Comunità Europee, 2005

ALSTON, P. (a cura di) Labour rights as human rights. Oxford: Oxford University Press. 2005

ANTONUCCI, M. Il campo di applicazione oggettivo e soggettivo della nuova normativa in materia di sicurezza e tutela della salute dei lavoratori. Diritto delle Relazioni Industriali n. 22008 p. 441-60

BARAK-EREZ D., GROSS A.M. (a cura di) Exploring social rights: Between theory and practice. Oxford; Portland, Oregon: Hart. 2007.

BARAM, M. Globalization and workplace hazards in developing nations. Safety Science, v. 37, 2009 p. 756-66. BAUMANN, A., et al. Mental health and well-being at the workplace: Protection and inclusion in challenging times. World Health Organization. 2010. Reperibile presso http://www.euro.who.int/_data/ assets/pdf_file/0018/124047/e94345.pdf (ultimo accesso 11 novembre 2010).

CHAMPLIN DELL P., KNOEDLER JANET T., (a cura di) The institutionalist tradition in Labor Economics. M.E. Sharpe Inc. 2004.

CLAPHAM, A. Human rights obligations of non-State actors. Oxford: Oxford University Press, 2006. COMITATO EUROPEO PER I DIRITTI SOCIALI. Digest of the Case Law of the European Committee of Social Rights, settembre 2008. Reperibile su <http://www.coe.int/t/dghl/monitoring/socialcharter/ Digest/DigestIndex_en.asp> Ultimo accesso 9 novembre 2010.

COMITATO EUROPEO PER I DIRITTI SOCIALI. Activity Report 2009. 25 June 2010. Reperibile su http://www.coe.int/t/dghl/monitoring/socialcharter/Presentation/ActivityReport2009_en.pdf. Ultimo accesso 9 novembre 2010 .

COMITATO ONU PER I DIRITTI ECONOMICI SOCIALI E CULTURALI. General Comment n. 14 (2000). E/C.12/2000/4. 11 agosto 2000.

COMMISSIONE DELLE COMUNITÀ EUROPEE. Libro Verde Promuovere un quadro europeo per la responsabilità sociale delle imprese. $\operatorname{COM}(2001) 366$ def. Bruxelles: 2001.

CONAGHAN, J. et al. (a cura di). Labour law in an era of globalization: Transformative practices \& possibilities. Oxford: Oxford University Press, 2002

CONFEDERAZIONE EUROPEA DEI SINDACATI (CES-ETUC). Occupational health and safety and environmental clauses in International Framework Agreements: A study. Reperibile su http://www.etuc. org/IMG/pdf/Global_Report-version_finale_anglaise-3.pdf. Ultimo accesso 9 novembre 2010.

CORBIZZI FATTORI, G., SIMONINI, F. Il benessere sul lavoro attraverso la valutazione dello stress lavorocorrelato: un convegno. ISL - Igiene e Sicurezza del Lavoro. n. 5 2010. p. 261 - 63.

CRAVEN M. C. R. The International Covenant on Economic Social and Cultural Rights: a perspective on its development. Oxford: Clarendon, 1995.

DAMIANI, L. INAIL e benessere organizzativo: nuove prospettive di tutela per i dipendenti. I diritti dell'uomo. n. 2 2008. p. 53 - 57.

DONNELLY, J. Universal Human Rights in theory and practice. Ithaca: Cornell University Press. 2002

DUPUY R. J. (a cura di) The right to health as a human right. Alpen aan den Rijn: Sijthoff \& Noordhhoff, 1979

FIDH - International Federation for Human Rights, Corporate Accountability for Human Rights Abuses: A guide for victims and NGOs on recourse mechanisms, 2010. Reperibile presso http://www.fidh.org/ Corporate-Accountability-for-Human-Rights-Abuses. Ultimo accessp 9 novembre 2010.

GALIZIA, C. Responsabilità sociale delle imprese ed autonomia collettiva. Diritti Lavori Mercati n. 12009 p. 191-219

GALLO CARRABBA, A. La progettazione del benessere nei luoghi di lavoro dal d. lgs. 626/94 ai modelli di wellness management. L'Amministrazione italiana. n. 4 2008. p. 487 - 507

GHAI, D. Decent work: Concept and indicators. International labour review. v. 142 n. 2 2003. p. 113-45

GROSS, J. A., COMPA, L. (a cura di) Human rights in labor and employment relations: international and domestic perspectives. Champaign, Ill: Labor and employment relations association, 2009.

HÄMÄLÄINEN, P. The effect of globalization on occupational accidents. Safety Science, v. 372009 p. 733 - 
42

HEPPLE, B. (a cura di). Social and labour rights in a global context: International and comparative perspectives. Cambridge: Cambridge University Press, 2002.

HEPPLE, B. Labour laws and global trade. Oxford; Portland, Or.: Hart Publishing, 2005

INTERNATIONAL TRADE UNION CONFEDERATION (ITUC). 2nd World Congress Resolution on extending social protection and ensuring good occupational health and safety. $2 \mathrm{CO} / \mathrm{E} / 6.12$ (final). Vancouver: 2010

LASAK, K. Health and safety in the European Social Charter. International Journal of Comparative Labour Law and Industrial Relations. v. 25 n. 2 2003. p. 109-22

LEVY FEITSHANS I. Occupational health as a human right. Reperibile su http://www.ilo.org/safework_ bookshelf/english?content\&nd=857170259 (ultimo accesso 23 settembre 2010).

MALZANI, F. Obbligo di sicurezza e nuove latitudini: lo stress da lavoro (Nota a Cassazione sezione lavoro 7 giugno 2007, n. 13309). La Nuova Giurisprudenza Civile Commentata. n. 1 2008. p. 34 - 39.

MANZEY, D., MAROLD, J. Editorial. Occupational accidents and safety: The challenge of globalization. Safety Science, v. 37 2009, p. 723-26

MAUPAIN, F. New foundation or new façade? The ILO and the 2008 Declaration on Social Justice for a fair globalization. The European Journal of International Law. vol. 20 n. 3. 2009. p. 823-52

MONTERO, M. J. et al. Occupational Health and Safety in the framework of corporate social responsibility. Safety Science vol. 472009 p. 1440-45

MONTUSCHI, L., TULLINI, P. (a cura di) Lavoro e Responsabilità Sociale d'Impresa. Bologna: Zanichelli, 2006

NAPOLI, M. (a cura di) Globalizzazione e rapporti di lavoro. Milano: Vita e Pensiero, 2006.

OLSEN, O. E., LINDØE, P. H., Risk on the ramble: The international transfer of risk and vulnerability. Safety Science vol. 472009 p. 743-55

ORGANIZZAZIONE INTERNAZIONALE DEL LAVORO. Health and life at work: a basic human right. 2009. Reperibile su http://www.ilo.org/public/portugue/region/eurpro/lisbon/pdf/28abril_09_en.pdf. Ultimo accesso 9 novembre 2010.

ORGANIZZAZIONE INTERNAZIONALE DEL LAVORO. Plan of action (2010-2016) to achieve widespread ratification and effective implementation of the occupational safety and health instruments (Convention No. 155, its 2002 Protocol and Convention No. 187). Adottato dal Comitato Esecutivo dell'OIL nel corso della sua 307ma sessione, nel marzo 2010. Reperibile presso http://www.ilo.org/ wcmsp5/groups/public/---ed_norm/---normes/documents/genericdocument/wcms_125616.pdf. Ultimo accesso 9 novembre 2010.

PARLAMENTO EUROPEO. Risoluzione del 23 maggio 2007 sulla promozione di un lavoro dignitoso per tutti (2006/2240(INI)) in Gazzetta Ufficiale dell'Unione Europea C 102 E/321 del 24 aprile 2008.

PASCUCCI, P. Il campo di applicazione soggettivo delle nuove regole sulla salute e sicurezza nei luoghi di lavoro in Diritti Lavori Mercati n. 22008 p. 297-338

PEDRAZZOLI, M. Verso un nuovo lessico giuslavoristico: inventario di questioni. Settembre 2009, inedito. PERULLI, A. (a cura di) L'impresa responsabile: Diritti sociali e Corporate Social Responsibility. Matelica: Halley, 2007

PERULLI, A., MARRELLA, F. (estensori) Manifesto di Venezia per la regolazione della globalizzazione economica: La globalizzazione virtuosa. Rivista Italiana di Diritto del Lavoro n. 32009 p. 341-48

PESSI, R. Lavoro, mercato, “ordine spontaneo", regolazione transnazionale. Rivista Italiana di Diritto del Lavoro n. 32009 p. 277-97

PESSI, R. Persona, lavoro e poteri privati. Argomenti di diritto del lavoro n. 32009 p. 675-90

SCARPONI, S. La "privatizzazione" delle norme internazionali di tutela del lavoro: codici etici di responsabilità sociale, accordi sindacali internazionali. Lavoro e Diritto n. 32009 p. 403-425

SENATO DELla REPUBbliCA, XVI Legislatura. Commissione Parlamentare di Inchiesta sul fenomeno degli infortuni sul lavoro con particolare riguardo alle cosiddette "morti bianche". Audizione di Rappresentanti dell'OIL. 42a seduta, martedì 26 gennaio 2010. Testo reperibile su http://www.senato.it/ commissioni/156058/156060/156062/277175/278191/schedasedutacommissione.htm (ultimo accesso 23 settembre 2010). 
SEPULVEDA, M.M. The nature of the obligations under the International Covenant on Economic, Social and Cultural rights. Antwerpen (etc.): Intersentia, 2003

TOEBES, B. C. A. The right to health as a human right in international law. Antwerpen (etc.): Intersentia, 1999

VALLEBONA, A. I contratti di lavoro. Tomo I. Torino: UTET giuridica; Milanofiori, Assago: Wolters Kluwer Italia, 2009.

VALLEBONA, A. "Caritas in Veritate” e diritto del lavoro. Rivista Italiana di Diritto del Lavoro n. 12009 p. $439-45$

WILPERT, B. Impact of globalization on human work. Safety Science, v. 37, p. 727-32 